Yan Zeng* and Chao-Jun Du

\title{
The crystal structure of 3,5-difluoroisonicotinic acid, $\mathrm{C}_{6} \mathrm{H}_{3} \mathrm{~F}_{2} \mathrm{NO}_{2}$
}

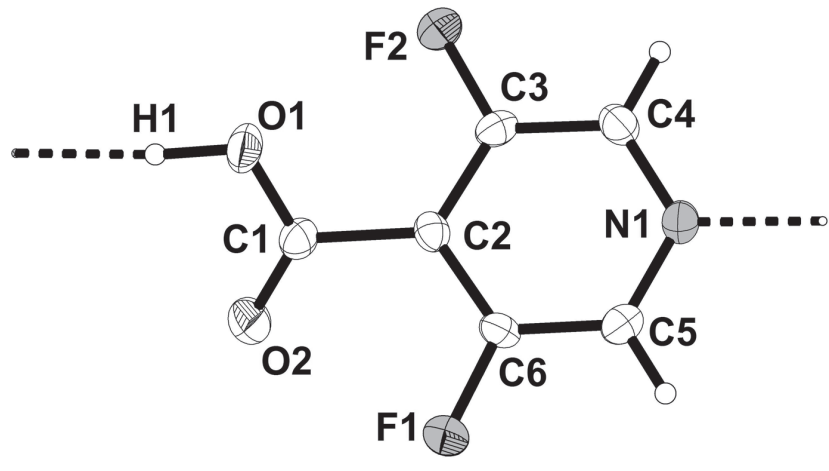

https://doi.org/10.1515/ncrs-2020-0144

Received March 16, 2020; accepted April 8, 2020; available online April 29, 2020

\section{Abstract}

$\mathrm{C}_{6} \mathrm{H}_{3} \mathrm{~F}_{2} \mathrm{NO}_{2}$, monoclinic, $P 2_{1} / n$ (no. 14), $a=6.8050(10) \AA$, $b=6.8443(9) \AA, \quad c=12.7196(14) \AA, \quad \beta=94.562(4)^{\circ}$, $V=590.54(13) \AA^{3}, Z=4, R_{\mathrm{gt}}(F)=0.0395, w R_{\text {ref }}\left(F^{2}\right)=0.1038$, $T=150(2) \mathrm{K}$.

\section{CCDC no.: 1995576}

The molecular structure is shown in the figure. Hydrogen bonds are shown as dashed lines. Table 1 contains crystallographic data and Table 2 contains the list of the atoms including atomic coordinates and displacement parameters.

\section{Source of material}

All of the starting materials were used as received. $1.59 \mathrm{~g}$ (0.01 mol) 3,5-difluoroisonicotinic acid was added to a solution mixed by $10 \mathrm{~mL}$ THF and $1 \mathrm{~mL}$ double-destilled water under room temperature. After stirring for $10 \mathrm{~min}$, the solution was filtered and let evaporate automatically. Many colorless block crystals were harvested, which are suitable for single-XRD measurement, yield 67.3\% (based on 3,5-difluoroisonicotinic acid).

*Corresponding author: Yan Zeng: College of Chemistry and Chemical Engineering, Xinxiang University, Xinxiang, Henan 453003, P.R. China, e-mail: zengyaner@126.com. https://orcid.org/00000001-7694-7434

Chao-Jun Du: School of Biochemical and Chemical Engineering, Nanyang Institute of Technology, Nanyang, Henan 473000, P.R. China. https://orcid.org/0000-0002-4767-7057
Table 1: Data collection and handling.

\begin{tabular}{ll}
\hline Crystal: & Colorless block \\
Size: & $0.25 \times 0.20 \times 0.15 \mathrm{~mm}$ \\
Wavelength: & Mo $K \alpha$ radiation $(0.71073 \AA)$ \\
$\mu:$ & $0.18 \mathrm{~mm}^{-1}$ \\
Diffractometer, scan mode: & Bruker APEX-II, $\varphi$ and $\omega$ \\
$\theta_{\max }$, completeness: & $26.0^{\circ},>99 \%$ \\
$N(h k l)_{\text {measured }}, N(h k l)_{\text {unique }}, R_{\text {int }}:$ & $10438,1158,0.062$ \\
Criterion for $I_{\text {obs }}, N(h k l)_{\text {gt }}:$ & $I_{\text {obs }}>2 \sigma\left(l_{\text {obs }}\right), 881$ \\
$N(\text { param })_{\text {refined }}:$ & 104 \\
Programs: & Bruker [1], SHELX [2], Olex2 [3, 4] \\
\hline
\end{tabular}

Table 2: Fractional atomic coordinates and isotropic or equivalent isotropic displacement parameters $\left(\AA^{2}\right)$.

\begin{tabular}{lrrrr}
\hline Atom & $\boldsymbol{x}$ & $\boldsymbol{y}$ & $\boldsymbol{z}$ & $\boldsymbol{U}_{\text {iso }} \boldsymbol{U}_{\text {eq }}$ \\
\hline C1 & $0.7052(3)$ & $0.2517(3)$ & $0.19376(14)$ & $0.0182(4)$ \\
C2 & $0.5748(3)$ & $0.2754(3)$ & $0.28316(13)$ & $0.0165(4)$ \\
C3 & $0.3854(3)$ & $0.3507(3)$ & $0.27058(13)$ & $0.0176(4)$ \\
C4 & $0.2684(3)$ & $0.3694(3)$ & $0.35407(14)$ & $0.0188(4)$ \\
H4 & 0.138722 & 0.420521 & 0.342231 & $0.023^{*}$ \\
C5 & $0.5192(3)$ & $0.2450(3)$ & $0.46777(13)$ & $0.0195(4)$ \\
H5 & 0.567202 & 0.206882 & 0.536910 & $0.023^{*}$ \\
C6 & $0.6397(3)$ & $0.2259(3)$ & $0.38566(14)$ & $0.0181(4)$ \\
F1 & $0.82217(16)$ & $0.15257(17)$ & $0.40713(8)$ & $0.0279(3)$ \\
F2 & $0.31187(15)$ & $0.41032(17)$ & $0.17503(8)$ & $0.0257(3)$ \\
N1 & $0.3367(2)$ & $0.3161(2)$ & $0.45141(11)$ & $0.0188(4)$ \\
O1 & $0.61250(19)$ & $0.1734(2)$ & $0.10957(9)$ & $0.0232(4)$ \\
H1 & $0.697(4)$ & $0.168(4)$ & $0.050(2)$ & $0.082(10)^{*}$ \\
O2 & $0.8764(2)$ & $0.3024(2)$ & $0.20209(10)$ & $0.0273(4)$ \\
\hline
\end{tabular}

\section{Experimental details}

The structure was solved by direct methods with the SHELXS program. All $\mathrm{H}$-atoms from $\mathrm{C}$ atoms were positioned with idealized geometry and refined isotropically $\left(U_{\text {iso }}(\mathrm{H})=1.2 U_{\text {eq }}(\mathrm{C})\right)$ using a riding model with $\mathrm{C}-\mathrm{H}=0.950 \AA$. The hydrogen atom of the carboxyl group was positioned on the basis of a $Q$ peak and refined freely.

\section{Comment}

It's known that fluorine-substituted isonicotinic acid derivatives have been used as ligands to construct metalorganic frameworks combined with transition metal and lanthanide, including 2-fluoroisonicotinic acid [5, 6] and 3-fluoroisonicotinic acid [7-10]. The same is true for 
3,5-dichloroisonicotinate containing complexes [11, 12]. However crystal structures of mono- or multi-fluorine-substituted have not been reported.

As shown in the figure, the title compound crystallizes with the whole organic molecule as its asymmetric unit. All atoms are nearly co-planar except the two $\mathrm{O}$ atoms and one $\mathrm{H}$ atom from the carboxyl group. The distances of N1 $\cdots \mathrm{H} 1$ and $01 \cdots \mathrm{H} 1$ are 1.636 and $0.99 \AA$, respectively, and the bond length of $\mathrm{C} 1-\mathrm{O} 1$ is much longer (1.313 $\AA$ ) than the bond length of $\mathrm{C} 1=\mathrm{O} 2(1.212 \AA)$, which indicates that the carboxyl group is protonated. There is an one-dimesional supramolecular structure generated by the $\mathrm{O}-\mathrm{H} \cdots \mathrm{N}$ hydrogen bond through a head-to-tail mode (see the figure).

\section{References}

1. Bruker. SAINT v8.37A. Bruker AXS Inc, Madison, WI, USA (2015).

2. Sheldrick, G. M.: Crystal structure refinement with SHELXL. Acta Crystallogr. C71 (2015) 3-8.

3. Bourhis, L. J.; Dolomanov, O. V.; Gildea, R. J.; Howard, J. A. K.; Puschmann, H.: The anatomy of a comprehensive constrained, restrained refinement program for the modern computing environment-Olex2 dissected. Acta Crystallogr. A71 (2015) 59-75.

4. Dolomanov, O. V.; Bourhis, L. J.; Gildea, R. J.; Howard, J. A. K.; Puschmann, H.: OLEX2: a complete structure solution, refinement and analysis program. J. Appl. Crystallogr. 42 (2009) 339-341.
5. Wang, X.-M.; Wu, W.-P.; Jiang, Y.-H.; Yang, G.-P.; Xi, Z.-P.: Six lanthanide-coordination polymers based on 2fluoroisonicotinic acid: synthesis, structure, luminescence and magnetic properties. Chin. J. Inorg. Chem. 30 (2014) 192-203.

6. Wen, G.-L.; Liu, D.-F.; Wang, F.-W.; Zhu, Q.-Y.; Wei, Y.-J.; Bao, X.; Huang, G.-G.; Shi, S.-S.: Two $\mathrm{Ag}(\mathrm{I}) / \mathrm{Cd}(\mathrm{II})$ coordination polymers of 2-fluoroisonicotinic acid: syntheses, structures and luminescent properties. J. Inorg. Organomet. Polym. Mater. 26 (2017) 799-805.

7. Fleming, J.; Kelley, J.; Peterson Jr, L. R.; Smith, M. D.; Loye, H.-C.: Tetra-aqua-bis(3-fluoro-pyridine-4-carboxylato-kN) zinc(II) dihydrate. Acta Crystallogr. E66 (2010) $\mathrm{m} 244-\mathrm{m} 245$.

8. Pachfule, P.; Chen, Y.; Jiang, J.; Banerjee, R.: Fluorinated metalorganic frameworks: advantageous for higher $\mathrm{H}_{2}$ and $\mathrm{CO}_{2}$ adsorption or not? Chem. Eur. J. 18 (2012) 688-694.

9. Pachfule, P.; Garai, B.; Banerjee, R.: Functionalization and isoreticulation in a series of metal-organic frameworks derived from pyridinecarboxylates. Inorg. Chem. 55 (2016) 7200-7205.

10. Muldoon, P. F.; Liu, C.; Miller, C. C.; Koby, S. B.; Jarvi, A. G.; Luo, T.-Y.; Saxena, S.; O’Keeffe, M.; Rosi, N. L.: Programmable topology in new families of heterobimetallic metal-organic frameworks. J. Am. Chem. Soc. 140 (2018) 6194-6198.

11. Li, X.-L.; Feng, K.-X.; Xin, L.-Y.: Crystal structure of catenapoly[diaqua-bis(3,5-dichloropyridine-4-carboxylato- $\mathrm{K}^{1} \mathrm{O}$ )-bis $\left(\mu_{2}-4,4^{\prime}\right.$-bipyridine- $\left.\mathrm{K}^{2} N: N^{\prime}\right)$ cobalt(II)], $\mathrm{C}_{22} \mathrm{H}_{16} \mathrm{Cl}_{4} \mathrm{CoN}_{4} \mathrm{O}_{6}$. Z. Kristallogr. NCS 234 (2019) 639-640.

12. Ling-Yun, X.; Zhou-Ru, J.; Rong-Rong, G.: Crystal structure of diaqua-bis(3,5-dichloroisonicotinato- $\kappa^{1} O$ )-bis(1,3-di(pyridin-4yl)propane- $\mathrm{K}^{1} N$ ) cobalt(II), $\mathrm{C}_{38} \mathrm{H}_{36} \mathrm{Cl}_{4} \mathrm{~N}_{6} \mathrm{O}_{6} \mathrm{Co}$. Z. Kristallogr. NCS 234 (2019) 495-497. 